\title{
Cloning of adriamycin-resistant related (arr) gene in an adriamycin-resistant L1210 variant
}

\author{
Jae-Ryong Kim ${ }^{1}$, Seong-Yong $\mathrm{Kim}^{1}$ and \\ Jung-Hye Kim ${ }^{1,2}$ \\ 1 Department of Biochemistry, College of Medicine, \\ Yeungnam University, Daegu, Korea \\ 2 Correspondig author \\ Accepted 1 July 1998
}

Abbreviations: arr, adriamycin resistant related; MDR, multidrug resistance; L1210AdR, adriamycin-resistant L1210 variant; DD-PCR, differential display polymerase chain reaction; RACE, rapid amplification of cDNA end; P-gp, permeability-glycoprotein

\begin{abstract}
A partial fragment of novel sequence (arr, adriamycinresistant related) was previously identified using the differential display (DD)-PCR technique with adriamycin-resistant L1210 variant (L1210AdR), which shows a typical multidrug resistant (MDR) phenotypes. The present research shows the isolation of full length arr cDNA sequence. To clone the full length cDNA of arr gene, DD-PCR fragments were subjected to 5'- and 3'-Rapid Amplification of cDNA End (RACE) method. The cloned arr cDNA consisted of 770 bases and contained an open reading frame of 153 bases, encoding a protein of 51 amino acid with the molecular mass of $4 \mathrm{kDa}$ by in vitro translation reactions. Nor-thern blot analysis showed that a 770 bases transcript arr gene was overexpressed in adriamycin-resistant L1210 variant, but not in the parent suggesting that the arr gene may be involved in the adriamycin-resistant phenotypes.
\end{abstract}

Keywords: MDR, arr, DD-PCR, RACE

\section{Introduction}

The appearance of drug resistant cells is one of the major limiting factor in cancer chemotherapy. To overcome the drug-resistance, understanding of the mechanisms to obtain the resistance is necessary. Especially, some drug resistant cells selected for one drug in vitro show the cross resistance to other antitumor drugs different in their structures and mechanisms of action. The broad spectrum resistance to cancer chemotherapy has been called multidrug resistance (MDR) (Kartner and Ling, 1989). Several researchers have investigated the mechanisms to drug resistance in cancer cells, including overexpression and amplification of Permeability-glycoprotein (P-gp) encoded by the mdr1 gene (Gros et al., 1986). P-gp is a transmembrane glycoprotein which acts as an ATP-dependent drug efflux pump and reduces the intracellular drug accumulation, thereby causing resistance to several antitumor drugs including adrimycin and vincristine (Gerlach et al., 1986). In addition to P-gp, various mechanisms related to antitumor drug resistance have been extensively studied such as expression of multidrug resistant-associated protein (MRP) (Cole et al., 1992), overproduction of detoxification factors such as glutathione, or its related enzyme, glutathione Stransferase (Schecter et al., 1992). The decreased expression of a DNA topoisomerase II (Wolverton et al., 1989) or increase of DNA topoisomerase I (Kotoh et al., 1994) has been reported in antitumor drug resistant cells. The expression of sorcin (V19), a 22 kDa calcium binding protein has been shown to increase in some multidrug-resistant cell lines (Meyers et al., 1987). These findings suggest that some proteins or genetic alterations are important in the development of antitumor drug resistance in cancer cells.

In the previous studies, we isolated and characterized antitumor drug resistant variants from mouse lymphoblastic leukemia L1210 cells; adriamycin-resistant (L1210AdR), vincristine-resistant (L1210VcR), and cisplatin-resistant (L1210Cis) variants(Kim et al., 1991; Kim and Kim, 1994; Kim et al., 1995; Kim et al., 1996). Several differentially expressed fragments were identified between adriamycinresistant $L 1210 A d R$ and parent $L 1210$ cells using the differential display polymerase chain reaction (DD-PCR) technique (Kim et al., 1995). Northern blot analysis with DD-PCR fragment (190 bp) as a probe showed that a $0.7 \mathrm{~kb}$ transcript was identified to be overexpressed 10 times higher in L1210AdR cells than in the parent cells, leading us to designate this fragment as arr (adriamycinresistant related) sequence (Kim et al., 1995). There was no similar sequence to a partial sequence of arr gene through a homology search in GenBank DNA sequence database. In this paper, we report the cloning of the full length cDNA of arr gene.

\section{Materials and Methods}

\section{Cell culture}

Adriamycin-resistant mouse lymphoblastic leukemia L1210 variant was isolated as described previously 
(Kim et al., 1991; Kim and Kim, 1994; Kim et al., 1995; Kim et al., 1996). L1210 and the drug-resistant variants were cultured in Fisher's medium containing $10 \%$ heatinactivated bovine serum (Hyclone) and $50 \mathrm{mg} / \mathrm{ml}$ of gentamycin. Adriamycin $\left(10^{-5} \mathrm{M}\right)$ was added to the medium to maintain the drug resistance phenotypes.

\section{Total RNA and mRNA preparation}

Total RNAs from the cells were isolated by acid guanidium thiocyanate/ phenol/chloroform extraction methods (Chomczynski and Sacchi, 1987). The mRNAs were purified using a Poly (A) Tract mRNA purification kit (Promega) or oligo-dT cellulose column chromatography (Sambrook et al., 1989).

\section{Cloning of the arr gene by the Rapid Amplification of CDNA Ends (RACE) method.}

The differentially expressed cDNA fragments were isolated from L1210AdR cells using DD-PCR as described previously (Kim et al., 1995). To clone the full sequence, RACE was carried out using a Marathone cDNA amplification kit (Clontech) according to instructions supplied from the manufacturer with minor modifications. Based on the sequence of the partial DD-PCR fragment, the primers were designed as follows: arrR (5'-CAGAGGCA TAAACTCATCAG-3') for 5'-RACE, arrF (5'-GTGTGCAA TGGTGGTTATAG-3') for 3'-RACE. First and second strands of cDNA were synthesized from the RNA extracted from the L1210AdR cells with random primers. The Marathone cDNA amplification kit contained primers for AP1 (5'-CCATCCTAATACGACTCACTATAGGGC-3'), AP2 (5'-ACTCACTATAGGGCTCGAGCGGC-3'), cDNA systhesis primers (5'-TTCTAGAATTCAGCGGCCGC $(\mathrm{T})_{30}$ NN), and cDNA adaptor (5'C T A A TA C G A C T C A C T A TA G G G C T C G AGCGGCCGCCCGGGCAGGT-3'). The PCR was carried out in a mixture containing $4 \mathrm{ml}$ adaptor-ligated cDNA, $10 \mathrm{mM}$ Tris- $\mathrm{HCl}$ ( $\mathrm{pH} 8.3$ ), $50 \mathrm{mM} \mathrm{KCl}, 1.5 \mathrm{mM}$ $\mathrm{MgCl}_{2}, 0.001 \%$ gelatin, $0.25 \mathrm{mM}$ dNTP, $1 \mathrm{mM}$ AP1 primer (for the adapter), $1 \mathrm{mM}$ arrF primer (for the 3'RACE) or arrR primer (for the 5'-RACE), and $2 U$ Taq DNA polymerase. The PCR cycles were initial denaturation for $1 \mathrm{~min}$ at $94^{\circ} \mathrm{C}$; 30 cycles of $10 \mathrm{sec}$ at $94^{\circ} \mathrm{C}, 15 \mathrm{sec}$ at $60^{\circ} \mathrm{C}$, and $30 \mathrm{sec}$ at $72^{\circ} \mathrm{C}$; and final extension for $5 \mathrm{~min}$ at $72^{\circ} \mathrm{C}$. To identify the amplified DNA fragments, DNAs in the gel were transfered to a nylon membrane and hybridized with a ${ }^{32} \mathrm{P}$-labeled arrR primer for detection of 5'-RACE fragments and with a ${ }^{32} \mathrm{P}$-labeled arrF primer for detection of $3^{\prime}$-RACE fragments. Amplified DNA fragments were isolated from gel slices using a Qiagen gel extraction kit and subcloned into a pGEM-T vector (Promega), and sequenced by the dideoxychain-termination method using a Sequenase v2.0 sequencing kit (USB). To select colonies containing arr-specific DNA fragments amplified by RACE, PCR was carried out using a forward primer (5'-GTTTTCCCAGTCACGACGTTGT-3'), a reverse primer (5'-TGGAATTGTGAGCGGATAAC-3') with vector and an arrR or arrF primer for 5'- or 3'-RACE, respectively.

\section{In vitro transcription and translation of cloned arr sequence.}

To determine the protein translated from the arr, in vitro transcription and translation were performed using pARR$\mathrm{R}$ plasmid DNA as a template. The arrR1 fragments containing SP6 and T7 promoter were amplified from pARR-R1 plasmids. The amplified DNA fragments were separated on an agarose gel and purified using a Qiagen gel extraction kit. RNA from the amplified DNA fragments was transcribed using a Riboprobe in vitro transcription system (Promega) and the size and integrity of transcribed RNA was examined by an agarose gel electrophoresis. In vitro translation reaction to the RNA transcribed from arrR was performed in volumes of $20 \mathrm{ml}$ of $1 \mathrm{mg}$ RNA, $10 \mathrm{ml}$ wheat-germ extract, $100 \mathrm{mM}$ potassium acetate, $10 \mathrm{mCi}{ }^{35} \mathrm{~S}$-methionine, and $80 \mathrm{mM}$ amino acid mixture without methionine at $25^{\circ} \mathrm{C}$ for $2 \mathrm{~h}$. In vitro translated proteins were separated on a $15 \%$ SDS-polyacrylamide gel. The gel was fixed in $50 \%$ methanol $/ 10 \%$ acetic acid, incubated in $1 \mathrm{M}$ sodium salicylate $(\mathrm{pH} 6.0)$ for 30 min, and exposed to an X-ray film.

\section{Northern and Southern blot analysis.}

For Northern blot analysis, total RNA was isolated from indicated cells as described above, and equal amount of RNA $(15 \mu \mathrm{g})$ was separated on $1.5 \%$ agarose gel containing $6.7 \%$ formaldehyde and MOPS buffer $(20 \mathrm{mM}$ MOPS, $5 \mathrm{mM}$ sodium acetate, and $1 \mathrm{mM}$ EDTA, pH 7.0), and trans-ferred to a Hybond $\mathrm{N}^{+}$membrane (Amersham). The membrane was hybridized with [ $\alpha-$ ${ }^{32} \mathrm{P}$ ]dCTP-labeled full size of arr at a $42^{\circ} \mathrm{C}$ in a solution containing $0.25 \mathrm{M} \mathrm{NaCl}, 1 \% \mathrm{SDS}, 50 \%$ formamide, $2 \times$ Denhardt's solution, $100 \mathrm{mg} / \mathrm{ml}$ salmon sperm DNA, and $5 \%$ dextran sulfate. Blots were washed under high stringency $(2 \times \mathrm{SSC} / 0.1 \%$ SDS at room temperature for $20 \mathrm{~min}$ followed by $0.5 \times \mathrm{SSC} / 0.1 \% \mathrm{SDS}$ at $50^{\circ} \mathrm{C} 2$ times for $30 \mathrm{~min}$ ). For Southern blot analysis, genomic DNA was isolated from indicated cells and digested with EcoRI. The DNA digests were electro-phoresed and transferred to a Hybond $\mathrm{N}^{+}$membrane. Hybridization was carried out with the same conditions as used in Northern blotting.

\section{Results and Discussion}

Previously, adriamycin-resistant variant (L1210AdR) was selected and characterized from mouse lymphoblastic leukemia L1210 cells (Kim et al., 1991; Kim and Kim, 1994; Kim et al., 1995; Kim et al., 1996). Using a DD- 
PCR technique, a partial 190-bp PCR fragment, named arr (adriamycin resistant related) sequence, was isolated from L1210AdR (Kim et al., 1995). Northern blot analysis revealed that $0.8 \mathrm{~kb}$ transcript could be observed in the total RNA fractions from L1210AdR cells, however, not detected in the poly $(\mathrm{A})$-RNA fractions using a probe with original 190-bp PCR fragment (data not shown). These findings imply that the transcript for arr gene does not contain the $\operatorname{poly}(A)$ tail. Thus, we tried to isolate the fulllength sequence of arr gene on cDNA library synthesized

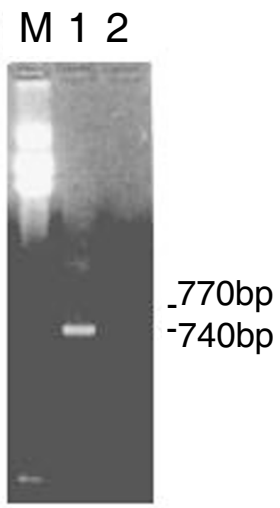

Figure 1. Identification of DNA fragments by 5'-RACE. PCR products were purified and amplified on a cDNA library, prepared from L1210AdR-derived total RNA with random primers (lane 1) or oligo-dT primers (lane 2). A nestde PCR was done with AP2 and ArrR primers $M$ indicates the 123 bp DNA ladder. from L1210AdR-derived total RNA using the RACE method.

ArrR primers were synthesized on the basis of the original DD-PCR fragments. The cDNA was synthesized from L1210AdR-derived total RNA using oligo(dT) or random primers. 5'-RACE was performed and then amplified by a nested PCR with AP2 and ArrR primers. An each aliquots of $\mathrm{PCR}$ reaction was used as the template for the second amplification. Only the second step of amplification allowed the detection of fragments, showing 770 and 740 base pairs in length, by ethidium bromide staining after gel electrophoresis (Figure 1). Amplified DNA fragments were isolated from gel slices using a Qiagen gel extraction kit, and subcloned into a pGEM-T vector. Plasmids containing DNA inserts of the expected size were selected for sequencing analysis.

The complete arr sequence was obtained by matching the overlapping sequences between original DD-PCR fragments and each PCR product (Figure 2). Sequence analysis of the cloned DNA fragments demonstrated that $770 \mathrm{bp}$ fragment contained the open reading frame of 156 base pairs encoding a polypeptide of 51 amino acids. The nucleotide sequence around the proposed initiation codon (ATG) is in agreement with the Kozak consensus sequence (Kozak, 1984). The deduced amino acid sequence of arr gene was not identical with any reported sequences in the data base and shared weak homology of less than $40 \%$.

The arr gene was observed at about $770 \mathrm{bp}$ in the total RNA fractions from L1210AdR cells, however, it was not detected in the poly(A)-RNA fractions using a probe with

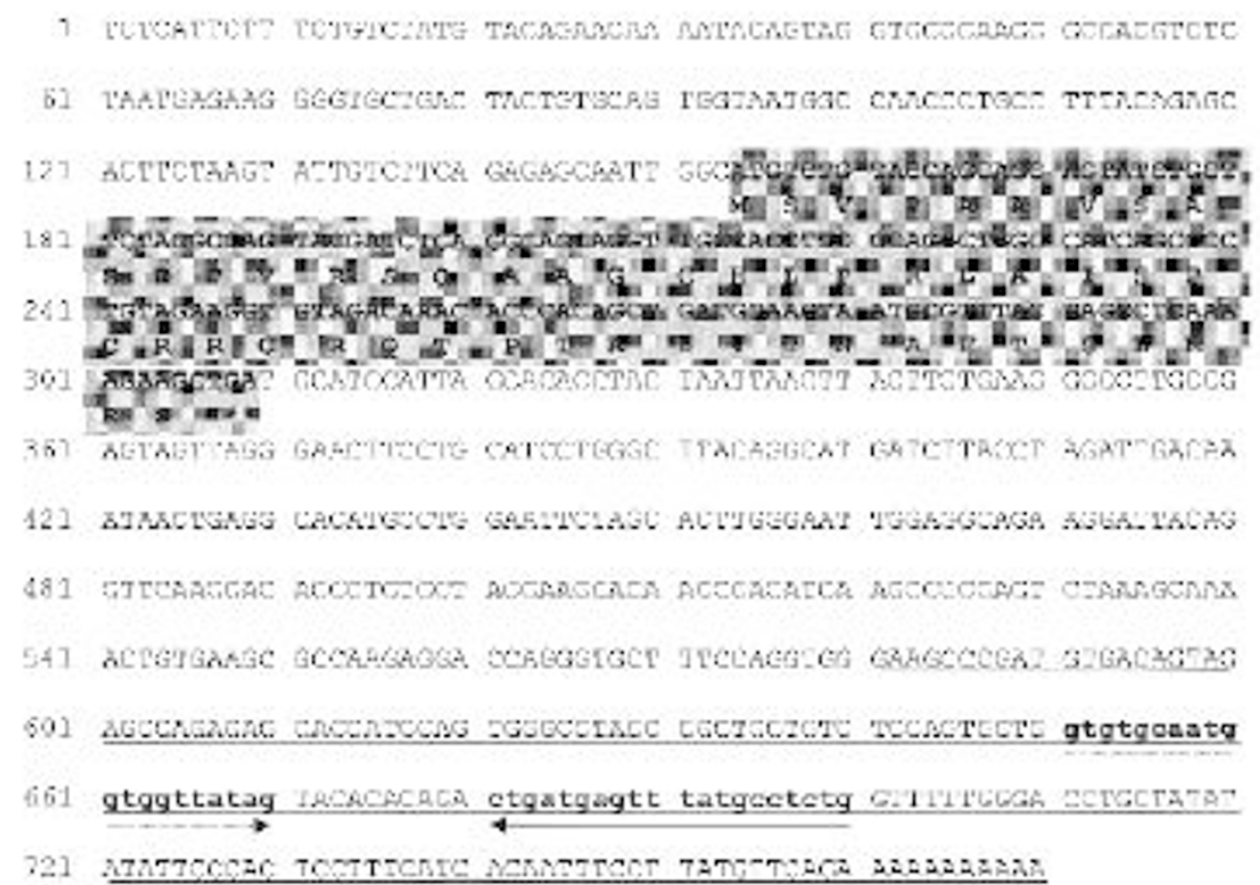

Figure 2. Nucleotide sequence and deduced amino acid sequence of arr. The nucleotide sequence data has been deposited in a GenBank with the accession number AF039839. The possible open reading frame is boxed, and the position of the stop codon is indicated by $* * *$. The oligonuclotide sequences used in the primer for 5'-RACE (arrR) or 3'-RASE (arrF)is indicated by solid arrow or dashed arrow, respectively. The original $190 \mathrm{bp}$ of DD-PCR fragment is underlined. 
original DD-PCR fragment or cloned ArrR (data not shown). In addition, a consensus polyadenylation signal (AAUAA) in arr sequence was not found. When the $3^{\prime}-$ RACE from the 3'-end was performed it was found that the 204 and 860 bp long fragments were yielded (data not shown). However, these fragments failed to detect 0.8 kb transcript in L1210AdR cells by Northern blot analysis (data not shown). From these findings, it is suggested that arr transcript does not contain the poly $(\mathrm{A})$ tail.

To define the protein translated from the arr sequence, in vitro transcription and translation were analyzed using pARR-R1 plasmid DNA containing T7 and SP6 promoter as a template. The arrR DNA was amplified from pARRR1 plasmid with forward and reverse primers, and transcribed with T7 or SP6 RNA polymerase by using a Riboprobe in vitro transcription system. As shown in Figure $3 \mathrm{~A}$, antisense T7 and sense SP6 transcripts were observed as the same size. However, only the sense transcript with SP6 RNA polymerase was translated to a polypeptide with a molecular mass of $4 \mathrm{kDa}$ in wheat germ extract (Figure 3B).

To determine the expression of the arr gene in the various drug resistant cells, Northern blot analysis was done. Equal amounts of total RNA isolated from L1210AdR variants (Kim et al., 1991; Kim and Kim, 1994; Kim et al., 1995; Kim et al., 1996) was separated, blotted and

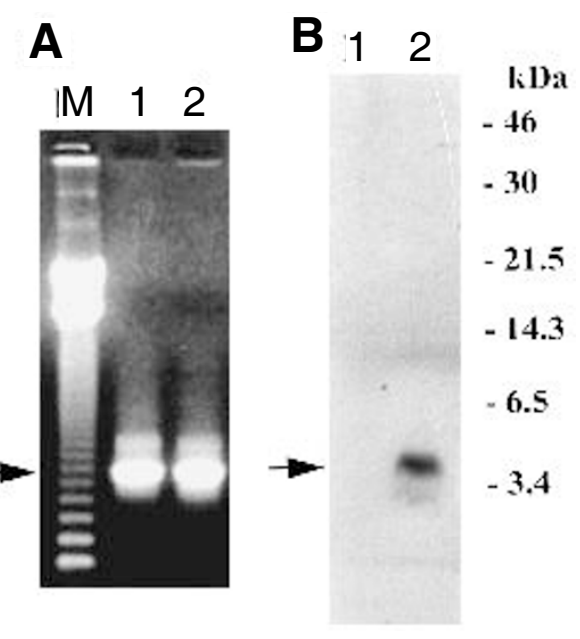

Figure 3. In vitro transcription (A) and in vitro translation (B) of arrRsequence. A. The arrRDNA containing $T 7$ and SP promotor was amplified from pARR-R1 with FP and RP primers and described with T7 or SP6 RNA polymerase using Riboprobe in vitro transcription system (Promega corp.). M, 123 ladder; 1, T7 transcripts of arrR containing antisense RNA sequence; 2, SP6 transcripts of arrR containing sense RNA sequence. B. The transcribed RNAs were translated with wheat germ extract and ${ }^{35} \mathrm{~S}$ methionine. The translated products were seperated in 15\% SDS-polyacrylamide gel. The gel was fixed, dried, and exposed to a X-ray film for 4 days. lane 1 ; The translated products from the $T 7$ transcript, lane 1 ; The translated products from the SP6 transcript hybridized with $32 \mathrm{P}$-labeled arrR. As shown in Figure 4A, The arr gene was detected as a single RNA species (770 bp) and highly expressed in L1210AdR, but not in the parent $L 1210$ cells. It was further confirmed by genomic Southern blot analysis. The Southern blotting pattern showed three distinct bands $(0.7,2.1$ and 2.9 $\mathrm{kb}$ ) were amplified in EcoRI fragments of L1210AdR as compared to those of parent L1210 cells (Figure 4B). These results suggested that the expression of arr gene appears to be closely associated with adriamycin and vincristine mediated drug resistance, however, it is unlikely to represent rearranged DNA amplification.

Permeability-glycoprotein (P-gp) encoded by the $m d r 1$ gene that functions as an ATP-dependent drug-efflux pump has been shown to confer resistance to several structurally unrelated drugs, including adriamycin, mitomycin, Vinca alkaloids, and other natural drugs (Fojo et al., 1985; Shen et al., 1986; Bradley et al., 1988). Amplification of the mdr1 gene has been observed in some solid tumors such as colorectal and renal-cell carcinomas (Fojo et al., 1987; Goldstein et al., 1989). In addition, the $m d r$ gene was highly expressed in $L 1210 \mathrm{VcR}$ and L1210AdR cells, but not detected in the parent L1210 and L1210Cis cells (Kim et al., 1991; Kim and Kim, 1994; Kim et al., 1995; Kim et al., 1996). Both L1210VcR and L1210AdR variants have exhibited typical multidrug resistant phenotypes; the cross resistance to plant alkaloids such as vinblastine and vincristine, etoposide, methotrexate, dactinomycin, daunorubicin and epirubicin (Kim et al., 1991; Kim and Kim, 1994; Kim et al., 1995; Kim et al., 1996).

At present, it remains unclear whether the increased expression of arr gene is important in the development

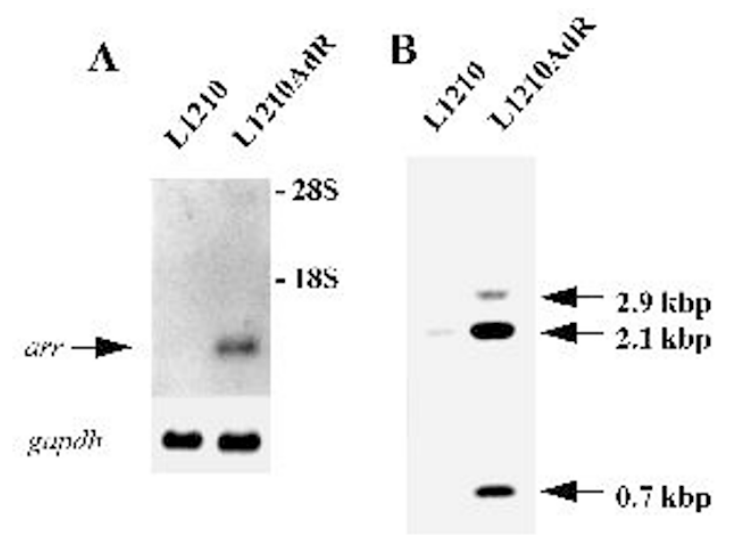

Figure 4. Northern and Southern blot analyses. Fifteen mg of total RNA (A) or five mg of EcoRI-digested genomic DNA (B) isolated from indicated cells was separated and blotted onto the Hybond $\mathrm{N}^{+}$membrane. Hybridization was carried out with the full-size of ${ }^{32} \mathrm{P}$-labeled arr. L1210; parent L1210 cells, L1210AdR; adriamycin-resistant L1210 variant. 
of antitumor drug resistance. However, it is probable that increased arr expression may be related to adriamycinresistant phenotypes. Regarding the absence of arr transcript in the mRNA fraction, additional studies will be necessary to determine the significance of the expression of arr gene for the development of multidrug resistance.

In summary, we isolated a novel sequence, named as arr, in adriamycin-resistant L1210 varient by using DDPCR and 5'-RACE methods. arr gene consists of 770 bases containing an open reading frame of 153 bases, which encodes a protein of 51 amino acid with the molecular mass of $4 \mathrm{kDa}$ by in vitro translation reactions. The expression of arr is enhanced in adriamycin-resistant L1210 variant.

\section{Acknowledgement}

This work was supported by a grant No. KOSEF 9510709-054-1 from the Korea Science and Engineering Foundation.

\section{References}

Bradley, G., Juranka, P. F. and Ling, V. (1988) Mechanism of multidrug resistance. Biochem. Biophys. Acta. 948: 87-128

Chomczynski, P. and Sacchi, N. (1987) Single-step method of RNA isolation by acid guanidinium thiocyanate-phenol-chloroform extraction. Anal. Biochem. 162: 156-159

Cole, S. P., Bhardwaj, G., Gerlach, J. H., Mackie, J. E., Grant, C. E., Almquist, K. C.,Stewart, A. J., Kurz, E. U., Duncan, A. M. and Deeley, R. G. (1992) Overexpression of a transporter gene in a multidrug-resistant human lung cancer cell line. Science 258 : 1650-1654

Fojo, A. T., Akiyama, S., Gottesman, M. M. and Pastan, I. (1985) Reduced drug accumulation in multiply drug-resistant human KB carcinoma cell lines. Cancer Res. 45 3002-3007

Fojo, A. T., Ueda, K., Slamon, D. J., Poplack, D. G., Gottesman, M. M. and Pastan, I. (1987) Expression of a multidrug-resistance gene in human tumors and tissues. Proc. Natl. Acad. Sci.USA 84: 265-269

Gerlach, J. H., Endicott, J. A., Juranka, P. E., Henderson, G., Sarangi, F., Deuchars, K L. and Ling, V. (1986) Homology between P-glycoprotein and a bacterial haemolysin transport protein suggests a model for multi-drug resistance. Nature 324: 485-489

Goldstein, L. J., Galski, H., Fojo, A. T., Willingham, M., Lai, S. L., Gazdar, A., Pirker, R. Green, A., Crist, W., Brodeur, G. M., Lieber, M., Cossman, J., Gottesman, M. M. and
Pastan, I. (1989) Expression of a multidrug resistance gene in human cancers. J. Nat. Cancer Inst. 81: 116-124

Gros, P., Neriah, Y. B., Croop, J. M. and Housman, D. E. (1986) Isolation and expression of a complementary DNA that confers multidrug resistance. Nature 323: 728731

Kartner, N. and Ling, V. (1989) Multidrug resistance in cancer. Sci. Am. 260: 26-33

Kim, J. H., Kim, J. R. and Lee, K. Y. (1991) Production of multidrug resistance by doxorubicin and its overcoming in mouse lymphoblastic leukemia L1210 cell. J. Korean Academy Arts. Sci. 30: 101-121

Kim, J. H., Kim, S. Y. and Kim, J. R. (1996) Effect of hyperthermia on anticancer drugresistant L1210 sublines. J. Korean Cancer Assoc. 28: 646-654

Kim, J. R. and Kim, J. H. (1994) A vincristine-resistant L1210 subline shows cross resistance, $m d r$ gene amplification and overexpression. Korean J. Biochem. 26: 67-76

Kim, J. R., Kim, S. Y. and Kim, J. H. (1995) Isolation of a novel sequence overexpressed and amplified in an adriamycin-resistant L1210 variant using differential display polymerase chain reaction. Korean J. Biochem.27: 149-155

Kotoh, S., Naito, S., Yokomizo, A., Kumazawa, J., Asakuno, K., Kohno, K. and Kuwano, M. (1994) Increased expression of DNA topoisomerase I gene and collateral sensitivity to camptothecin in human cisplatin-resistant bladder cancer cells. Cancer Res. 54: 32483252

Kozak, M. (1984) Compilation and analysis of sequences upstream from the translational start site in eukaryotic mRNAs. Nucleic Acids Res. 12: 857-872

Meyers, M. B., Schneider, K. A., Spengler, B. A., Chang, T. and Biedler, J. L. (1987) Sorcin (V19), a soluble acidic calcium-binding protein over-produced in multidrugresistant cells. Identification of the protein by anti-sorcin antibody. Biochem. Pharmacol. 35: $2373-2380$

Sambrook, J., Fritsch, E. F. and Maniatis, T. (1989) In Molecular Cloning, pp. 10.1410.17, Cold Spring Harbor Press, Cold Spring Harbor, NY

Schecter, R. L., Alaoui-Jamali, M. A. and Batist, G. (1992) Glutathione S-transferase in chemotherapy resistance and in carcinogenesis. Biochem. Cell Biol. 70: 349-353

Shen, D. W., Cardarelli, C., Hwang, J., Cornwell, M., Richert, N., Ishii S., Pastan, I. and Gottesman, M. M. (1986) Multiple drug-resistant human KB carcinoma cells independently selected for high-level resistance to colchicine, adriamycin, or vinblastine show changes in expression of specific proteins. J. Biol. Chem. 261: 7762-7770

Wolverton, J. S., Danks, M. K., Schmidt, C. A. and Beck, W. T. (1989) Genetic characterization of the multidrug-resistant phenotype of VM-26-resistant human leukemic cells. Cancer Res. 49: 2422-2426 